\title{
A single quenching point for a fractional heat equation based on the Riemann-Liouville fractional derivative with a nonlinear concentrate source
}

\author{
Wannika Sawangtong ${ }^{1,3}$ and Panumart Sawangtong ${ }^{2^{*}}$ (1)
}

"Correspondence:
panumart.s@sci.kmutnb.ac.th
2Department of Mathematics,
Faculty of Applied Science, King
Mongkut's University of Technology
North Bangkok, Pracharat 1 Road,
Bangkok, 10800, Thailand
Full list of author information is
available at the end of the article

\begin{abstract}
This paper aims to study the quenching problem in a fractional heat equation with the Riemann-Liouville fractional derivative. The existence and uniqueness of a solution for the problem are obtained by transforming the problem to an equivalent integral equation. The condition for the quenching occurrence in a finite time is given. Furthermore, the quenching point set is shown.
\end{abstract}

Keywords: quenching; fractional heat equation; Riemann-Liouville derivative; existence and uniqueness

\section{Introduction}

The study of quenching problems for the initial-boundary value problem of parabolic type began in 1975 with a research paper of Kawarada [1], and since then, this topic has attracted much attention. Recently, many papers have studied quenching problems in PDEs (see [24] and [5] etc.).

The purpose of this article is to investigate the quenching phenomenon of the following fractional heat equation based on the Riemann-Liouville fractional derivative with nonlinear concentrated source:

$$
\begin{aligned}
& v_{t}={ }_{\mathrm{RL}} D_{t}^{1-\alpha} v_{x x}+\delta\left(x-x_{0}\right) f\left(1-v\left(x_{0}, t\right)\right) \text { for }(x, t) \in(0, l) \times(0, \infty), \\
& v(x, 0)=0 \quad \text { for } x \in[0, l], \\
& v(0, t)=v(l, t)=0 \quad \text { for } t>0,
\end{aligned}
$$

where ${ }_{\mathrm{RL}} D_{t}^{1-\alpha}$ denotes the Riemann-Liouville fractional derivative of order $1-\alpha$ with $\alpha \in$ $(0,1), \delta(x)$ is the Dirac delta function, $x_{0}$ is a fixed point in $(0, l), l$ is a positive constant, and $f$ is a given function such that $f(1)>0$ and $f(1-v), f_{v}(1-v), f_{v v}(1-v)$ are positive for $0<v<1$ and $\lim _{v \rightarrow 1^{-}} f(1-v)=\infty$.

When $\alpha=1$, equation (1) becomes the heat equation with the homogeneous initial and boundary conditions. It is well known that the highly localized nature of the heat source can be represented by the Dirac delta function. In the case of $\alpha=1$, the model for the

(c) The Author(s) 2017. This article is distributed under the terms of the Creative Commons Attribution 4.0 International License (http://creativecommons.org/licenses/by/4.0/), which permits unrestricted use, distribution, and reproduction in any medium, provided you give appropriate credit to the original author(s) and the source, provide a link to the Creative Commons license, and indicate if changes were made. 
temperature of a diffusive medium with a nonlinear concentrated quenching source that moves at a constant speed was studied by Olmstead and Roberts [3] by analyzing the corresponding Volterra integral equation at the point $x_{0}=c t$, where $c>0$ is the constant speed of translation

$$
v\left(x_{0}, t\right)=\frac{1}{2(\pi t)^{\frac{1}{2}}} \int_{-\infty}^{\infty} e^{\frac{-(x-\xi)^{2}}{4 t}} v_{0}(\xi) d \xi+\int_{0}^{\infty} \frac{e^{\frac{-\left(x-x_{0}\right)^{2}}{4(t-s)}}}{2[\pi(t-s)]^{\frac{1}{2}}} f\left(1-v\left(x_{0}, s\right)\right) d s
$$

and the nonhomogeneous initial condition is determined by $v_{0}$. Under appropriate conditions, they proved that quenching always occurs for a sufficiently slow speed. Moreover, when quenching does occur, bounds for quenching time are shown. The research paper of Olmstead and Roberts [3] is inspiration and motivation for working and writing this article. We next introduce the quenching definition.

Definition 1.1 A solution $v$ of problem (1) is said to quench at the point $x_{0}$ if there exists a positive time $t_{q}$ such that the function $v\left(x_{0}, t\right) \rightarrow 1^{-}$and $v_{t}\left(x_{0}, t\right) \rightarrow \infty$ as $t \rightarrow t_{q}$. If $t_{q}$ is finite, then $v$ is said to quench in a finite time. On the other hand, if $t_{q}=\infty$, then $v$ is said to quench in an infinite time. Furthermore, the set of all quenching points is called the quenching set.

For the reader's convenience, we introduce some facts about fractional calculus. For details, one can see [6].

Definition 1.2 The integral

$$
I_{t}^{\alpha} f(t)=\frac{1}{\Gamma(\alpha)} \int_{0}^{t}(t-s)^{\alpha-1} f(s) d s
$$

is called the Riemann-Liouville fractional integral of order $\alpha>0$, where $\Gamma$ is the gamma function.

Definition 1.3 The Riemann-Liouville fractional derivative of order $\alpha$ for a function $f$ is defined by

$$
{ }_{\mathrm{RL}} D_{t}^{\alpha} f(t)=\frac{1}{\Gamma(n-\alpha)}\left(\frac{d}{d t}\right)^{n} \int_{0}^{t}(t-s)^{n-\alpha-1} f(s) d s \quad \text { for } n=[\alpha]+1,
$$

where $[\alpha]$ denotes the integer part of the real number $\alpha$.

Definition 1.4 For a function $f:[0, \infty) \rightarrow \mathbb{R}$, the Caputo derivative of fractional order $\alpha$ is defined as

$$
{ }_{\mathrm{C}} D_{t}^{\alpha} f(t)=\frac{1}{\Gamma(n-\alpha)} \int_{0}^{t}(t-s)^{n-\alpha-1} f^{(n)}(s) d s \quad \text { for } n=[\alpha]+1
$$

provided that such integral exists.

Lemma 1.1 Let $f$ be a continuous function on $[0, a]$ with $a>0$ and $f(0)=0$, and let $\alpha \in$ $(0,1)$, then 
(1) $I_{t}^{\alpha}\left({ }_{\mathrm{RL}} D_{t}^{\alpha} f(t)\right)=f(t)$,

(2) ${ }_{\mathrm{C}} D_{t}^{\alpha} f(t)=I_{t}^{1-\alpha} \frac{d}{d t} f(t)$.

In Section 2, we transform problem (1) into the equivalent Volterra integral equation. A unique nonnegative local solution of problem (1) is shown in Section 3. In Section 4, we prove that a solution of problem (1) quenches in a finite time. In the last section, we find the quenching set of problem (1).

\section{An integral equation}

To investigate a possible quenching solution of problem (1), it is advantageous to convert the initial-boundary problem (1) into an associated integral equation by the technique in [7]. To do so, we first find the Green's function $G_{\alpha}$ equivalent to problem (1) determined by the following system: for any fixed $(\xi, \tau) \in(0, l) \times(0, \infty)$,

$$
\begin{aligned}
& \frac{\partial G_{\alpha}}{\partial t}={ }_{\mathrm{RL}} D_{t}^{1-\alpha} \frac{\partial^{2} G_{\alpha}}{\partial x^{2}}+\delta(x-\xi) \delta(t-\tau), \\
& G_{\alpha}(0, t ; \xi, \tau)=0=G_{\alpha}(l, t ; \xi, \tau), \\
& G_{\alpha}(x, t ; \xi, \tau)=0 \quad \text { for } t<\tau .
\end{aligned}
$$

As shown in [8], the Green's function $G_{\alpha}$ can be expressed in terms of $G_{1}$, which is the Green's function for the classical diffusion in the case of $\alpha=1$

$$
G_{\alpha}(x, t ; \xi, \tau)=\int_{0}^{\infty} f_{\alpha}(z) G_{1}\left(x,(t-\tau)^{\alpha} z ; \xi, 0\right) d z
$$

with

$$
f_{\alpha}(z)=\sum_{j=0}^{\infty} \frac{(-1)^{j} z^{j}}{j ! \Gamma(1-\alpha-\alpha j)} \geq 0 \quad \text { for } z \geq 0
$$

and

$$
G_{1}(x, t ; \xi, \tau)=\frac{2}{l} \sum_{n=1}^{\infty} \sin \frac{n \pi x}{l} \sin \frac{n \pi \xi}{l} e^{-\frac{n^{2} \pi^{2}}{l^{2}}(t-\tau)} \text { for } t>\tau .
$$

By (3) and (4), we obtain the following alternative forms for

$$
G_{\alpha}(x, t ; \xi, \tau)=\frac{2}{l} \sum_{n=1}^{\infty} \sin \frac{n \pi x}{l} \sin \frac{n \pi \xi}{l} \int_{0}^{\infty} f_{\alpha}(z) e^{-\frac{n^{2} \pi^{2}}{l^{2}}(t-\tau)^{\alpha} z} d z \quad \text { for } t>\tau .
$$

By a technique similar to the one used in [7], the integral equation related to (1) is given for any $(x, t) \in[0, l] \times[0, \infty)$ by

$$
v(x, t)=\int_{0}^{t} G_{\alpha}\left(x, t-\tau ; x_{0}, 0\right) f\left(1-v\left(x_{0}, \tau\right)\right) d \tau .
$$

To analyze equation (5), it is essential to know the properties of $G_{\alpha}$. Various properties of $G_{\alpha}$ can be derived from (2). By [9], it is seen that $G_{\alpha}$ is positive, $G_{\alpha}$ is continuously differentiable for $t>0, G_{\alpha}$ is decreasing with respect to $t, \int_{0}^{t} G_{\alpha}\left(x, t-\tau ; x_{0}, 0\right) d \tau$ is positive and $\frac{d}{d t} \int_{0}^{t} G_{\alpha}\left(x, t-\tau ; x_{0}, 0\right) d \tau$ is positive. 


\section{Existence and uniqueness}

In this section, we use the Banach fixed point theorem to show that the integral equation (5) has a unique continuous solution on the time interval $\left[0, t_{1}\right]$ for some $t_{1}>0$.

Theorem 3.1 There exists $t_{1}>0$ such that the integral equation (5) has a unique nonnegative continuous solution $v$ on the interval $\left[0, t_{1}\right]$ for any $x \in[0, l]$.

Proof Let $R$ be any fixed positive constant with $R<1$. We choose a positive constant $h$ satisfying the following inequality: for any $x \in[0, l]$,

$$
\int_{0}^{h} G_{\alpha}\left(x, h-\tau ; x_{0}, 0\right) d \tau<\min \left\{\frac{R}{f(1-R)}, \frac{1}{f_{v}(1-R)}\right\} .
$$

We construct the set $S_{R}$ by

$$
S_{R}=\left\{v \in C([0, l] \times[0, h]) \text { such that } \sup _{(x, t) \in[0, l] \times[0, h]}|v(x, t)| \leq R\right\} .
$$

Thus, $S_{R}$ is the nonempty closed convex subset of $C([0, l] \times[0, h])$. Then $S_{R}$ is a Banach space and its norm $\|v\|_{S_{R}}=\sup _{(x, t) \in[0, l] \times[0, h]}|v(x, t)|$. We next define an operator $F: S_{R} \rightarrow$ $C([0, l] \times[0, h])$ by

$$
F v(x, t)=\int_{0}^{t} G_{\alpha}\left(x, t-\tau ; x_{0}, 0\right) f\left(1-v\left(x_{0}, \tau\right)\right) d \tau
$$

for any $v \in S_{R}$. In order to apply the Banach fixed point theorem, we have to show that the operator $F$ satisfies the following two conditions:

(1) $F: S_{R} \rightarrow S_{R}$ and

(2) $F$ is a contraction mapping.

Condition (1): Let $v \in S_{R}$. By $\frac{d}{d t} \int_{0}^{t} G_{\alpha}\left(x, t-\tau ; x_{0}, 0\right) d \tau>0$ and (6), we have

$$
\begin{aligned}
|F v(x, t)| & \leq f(1-R) \int_{0}^{t} G_{\alpha}\left(x, t-\tau ; x_{0}, 0\right) d \tau \\
& \leq f(1-R) \int_{0}^{h} G_{\alpha}\left(x, h-\tau ; x_{0}, 0\right) d \tau \\
& \leq R
\end{aligned}
$$

This means that $\|v\|_{S_{R}}<R$.

Condition (2): Let us consider that for any $v, w \in S_{R}$,

$$
|F v(x, t)-F w(x, t)| \leq f_{v}(1-R) \int_{0}^{h} G_{\alpha}\left(x, h-\tau ; x_{0}, 0\right) d \tau\|v-w\|_{S_{R}} .
$$

By (6), this implies that $F$ is a contraction mapping.

Therefore, the Banach fixed point theorem implies that $F$ has a unique fixed point on $S_{R}$. We can conclude that the integral equation (5) has a unique continuous solution $v$ for any $(x, t) \in[0, l] \times[0, h]$. It follows by the positivity of $G_{\alpha}$ and $f$ that the solution $v$ is positive. Hence, the proof of this theorem is completed. 


\section{Finite time quenching}

The following theorem shows that the solution $v$ of the integral equation (5) quenches at the point $x_{0}$.

Theorem 4.1 Let $\left[0, t_{q}\right)$ be the maximum time interval such that the integral equation (5) has a continuously differentiable solution $v(x, t)$ for any $(x, t) \in[0, l] \times\left[0, t_{q}\right)$. If $v\left(x_{0}, t\right)$ converges to $1^{-}$as $t$ converges to $t_{q}$, then $v_{t}\left(x_{0}, t\right)$ goes to $\infty$ as $t \rightarrow t_{q}$.

Proof Let us consider the derivative of (5): for any $(x, t) \in(0, l) \times\left(0, t_{q}\right)$,

$$
\begin{aligned}
v_{t}(x, t)= & G_{\alpha}\left(x, t ; x_{0}, 0\right) f(1) \\
& +\int_{0}^{t} G_{\alpha}\left(x, t-\tau ; x_{0}, 0\right) f_{v}\left(1-v\left(x_{0}, \tau\right)\right) v_{\tau}(x, \tau) d \tau .
\end{aligned}
$$

From the positivity of $G_{\alpha}$ and the properties of $f$, we have that

$$
v_{t}(x, t)>0 \quad \text { for }(x, t) \in(0, l) \times\left(0, t_{q}\right)
$$

Equation (7):

$$
\begin{aligned}
v_{t}\left(x_{0}, t\right) & \geq G_{\alpha}\left(x_{0}, t ; x_{0}, 0\right)\left[f(1)+\int_{0}^{t} f_{v}\left(1-v\left(x_{0}, \tau\right)\right) v_{\tau}\left(x_{0}, \tau\right) d \tau\right] \\
& =G_{\alpha}\left(x_{0}, t ; x_{0}, 0\right) f\left(1-v\left(x_{0}, t\right)\right) .
\end{aligned}
$$

Since $\lim _{v \rightarrow 1^{-}} f(1-v)=\infty$, this shows that $v_{t}\left(x_{0}, t\right) \rightarrow \infty$ as $t \rightarrow t_{q}$. Therefore, we can conclude that $v$ quenches at the point $x_{0}$ when $t \rightarrow t_{q}$.

We next give the condition that guarantees the occurrence of quenching in a finite time.

Theorem 4.2 There exists a finite time $t^{*}$ such that the integral equation (5) has no continuous nonnegative solutions $v$ with $v(x, t)<1$ for every $x \in[0, l]$ and for $t>t^{*}$.

Proof Suppose that the integral equation (5) has a continuous solution $v$ with $v(x, t)<1$ for any $(x, t) \in[0, l] \times\left[0, t_{2}\right]$, where $t_{2}$ is determined later. Without loss of generality, let us consider integral (5) at the point $x=x_{0}$

$$
v\left(x_{0}, t\right)=\int_{0}^{t} G_{\alpha}\left(x_{0}, t-\tau ; x_{0}, 0\right) f\left(1-v\left(x_{0}, \tau\right)\right) d \tau
$$

for $t \in\left[0, t_{2}\right]$. We then choose $t_{2}$ where $t_{2}$ satisfies

$$
\int_{0}^{t_{2}} G_{\alpha}\left(x_{0}, t_{2}-\tau ; x_{0}, 0\right) d \tau \geq \int_{0}^{1} \frac{1}{f(1-r)} d r .
$$

Since $G_{\alpha}$ is decreasing with respect to $t$, we obtain that

$$
v\left(x_{0}, t\right)=\int_{0}^{t} G_{\alpha}\left(x_{0}, t-\tau ; x_{0}, 0\right) f\left(1-v\left(x_{0}, \tau\right)\right) d \tau \geq J(t)
$$


with $J(t)=\int_{0}^{t} G_{\alpha}\left(x_{0}, t_{2}-\tau ; x_{0}, 0\right) f\left(1-v\left(x_{0}, \tau\right)\right) d \tau$. We then have that

$$
\begin{aligned}
\frac{d J(t)}{d t} & =G_{\alpha}\left(x_{0}, t_{2}-t ; x_{0}, 0\right) f\left(1-v\left(x_{0}, t\right)\right) \\
& \geq G_{\alpha}\left(x_{0}, t_{2}-t ; x_{0}, 0\right) f(1-J(t))
\end{aligned}
$$

for any $t \in\left[0, t_{2}\right]$. Then, since $J\left(t_{2}\right)=v\left(x_{0}, t\right)<1$, we have

$$
\int_{0}^{1} \frac{1}{f(1-r)} d r>\int_{0}^{J\left(t_{2}\right)} \frac{1}{f(1-J(t))} d J \geq \int_{0}^{t_{2}} G_{\alpha}\left(x_{0}, t_{2}-t ; x_{0}, 0\right) d t
$$

which contradicts the definition of $t_{2}$. Therefore, this theorem is proved completely.

\section{Single quenching point}

The next theorem shows that the point $x_{0}$ is the single quenching point.

Theorem 5.1 Assume that the solution $v(x, t)$ of problem $(1)$ attains, for $t \in\left(0, t_{q}\right)$, its maximum at $\left(x_{0}, t\right)$. If $v\left(x_{0}, t\right)$ converges to $1^{-}$as $t$ approaches to $t_{q}$, then $v$ quenches at $x_{0}$. Moreover, $x_{0}$ is the single quenching point.

Proof It follows from Theorem 4.1 that $v$ quenches at the point $x_{0}$. We next show that $v$ quenches only at the point $x_{0}$. Since $v\left(x_{0}, t\right)$ exists for $t \in\left(0, t_{q}\right)$, we set $\omega(t)=v\left(x_{0}, t\right)$ for $t \in\left(0, t_{q}\right)$. Then problem (1) is equivalent to the following two initial-boundary value problems:

$$
\begin{aligned}
& \frac{\partial v}{\partial t}={ }_{\mathrm{RL}} D_{t}^{1-\alpha} \frac{\partial^{2} v}{\partial x^{2}} \quad \text { for }(x, t) \in\left(0, x_{0}\right) \times\left(0, t_{q}\right), \\
& v(x, 0)=0 \quad \text { for } 0 \leq x \leq x_{0}, \\
& v(0, t)=0 \text { and } \quad v\left(x_{0}, t\right)=\omega(t) \quad \text { for } 0<t<t_{q},
\end{aligned}
$$

and

$$
\begin{aligned}
& \frac{\partial v}{\partial t}={ }_{\mathrm{R} L} D_{t}^{1-\alpha} \frac{\partial^{2} v}{\partial x^{2}} \quad \text { for }(x, t) \in\left(x_{0}, l\right) \times\left(0, t_{q}\right), \\
& v(x, 0)=0 \quad \text { for } x_{0} \leq x \leq l, \\
& v\left(x_{0}, t\right)=\omega(t) \text { and } \quad v(l, t)=0 \text { for } 0<t<t_{q} .
\end{aligned}
$$

By taking the Riemann-Liouville fractional integral of order $1-\alpha$ on both sides of equations (9) and (10) and using Lemma 1.1, we obtain

$$
\begin{aligned}
& { }_{C} D_{t}^{\alpha} v(x, t)=\frac{\partial^{2} v}{\partial x^{2}} \quad \text { for }(x, t) \in\left(0, x_{0}\right) \times\left(0, t_{q}\right), \\
& v(x, 0)=0 \text { for } 0 \leq x \leq x_{0}, \\
& v(0, t)=0 \text { and } \quad v\left(x_{0}, t\right)=\omega(t) \quad \text { for } 0<t<t_{q},
\end{aligned}
$$


and

$$
\begin{aligned}
& { }_{C} D_{t}^{\alpha} v(x, t)=\frac{\partial^{2} v}{\partial x^{2}} \text { for }(x, t) \in\left(x_{0}, l\right) \times\left(0, t_{q}\right), \\
& v(x, 0)=0 \text { for } x_{0} \leq x \leq l, \\
& v\left(x_{0}, t\right)=\omega(t) \text { and } \quad v(l, t)=0 \text { for } 0<t<t_{q} .
\end{aligned}
$$

By the weak maximum principle for the Caputo fractional derivative [10], $v$ attains its maximum at the point $x_{0}$ for each of problems (11) and (12). Since $v$ is an increasing function of $t$ for $t \in\left(0, t_{q}\right)$, this implies that ${ }_{C} D_{t}^{\alpha} v(x, t)>0$. Then, for any $x \in\left(0, x_{0}\right)$, $\frac{\partial^{2} v}{\partial x^{2}}={ }_{C} D_{t}^{\alpha} v(x, t)>0$. Similarly, for any $x \in\left(x_{0}, l\right), \frac{\partial^{2} v}{\partial x^{2}}={ }_{C} D_{t}^{\alpha} v(x, t)>0$. Hence, $v$ is concave up for $x \in\left(0, x_{0}\right) \cup\left(x_{0}, l\right)$. Since $v$ attains its maximum at $\left(x_{0}, t\right)$ for $t \in\left(0, t_{q}\right)$ and $v$ is concave up for $x \in\left(0, x_{0}\right) \cup\left(x_{0}, l\right)$, this means that there is only a point $x_{0}$ such that $v\left(x_{0}, t\right)$ converges to $1^{-}$and $v_{t}\left(x_{0}, t\right)$ goes to $\infty$ as $t$ approaches to $t_{q}$. Hence, $x_{0}$ is the single quenching point.

\section{Acknowledgements}

This research was funded by The Thailand Research Fund (MRG5980119).

\section{Competing interests}

The authors declare that they have no competing interests.

\section{Authors' contributions}

PS formulated the research problem and wrote the paper. PS and WS participated in the derivation of the mathematical results. Both authors read and approved the final manuscript.

\section{Author details}

${ }^{1}$ Department of Mathematics, Faculty of Science, Mahidol University, 272 Rama VI Road, Bangkok, 10400, Thailand. ${ }^{2}$ Department of Mathematics, Faculty of Applied Science, King Mongkut's University of Technology North Bangkok, Pracharat 1 Road, Bangkok, 10800, Thailand. ${ }^{3}$ Centre of Excellence in Mathematics, PERDO, Commission on Higher Education, Ministry of Education, Bangkok, 10400, Thailand.

\section{Publisher's Note}

Springer Nature remains neutral with regard to jurisdictional claims in published maps and institutional affiliations.

Received: 28 December 2016 Accepted: 19 June 2017 Published online: 29 June 2017

\section{References}

1. Kawarada, $\mathrm{H}$ : On the solutions of initial boundary value problems for $u_{t}=u_{x x}+\frac{1}{(1-u)}$. Publ. Res. Inst. Math. Sci. 10, 729-736 (1975)

2. Kirk, CM, Roberts, CA: A quenching problem for the heat equation. J. Integral Equ. Appl. 14, 53-72 (2002)

3. Olmstead, WE, Roberts, CA: Critical speed for quenching. Dyn. Contin. Discrete Impuls. Syst., Ser. A Math. Anal. 8, 77-88 (2001)

4. Roberts, CA, Olmstead, WE: Local and non-local boundary quenching. Math. Methods Appl. Sci. 22, 1465-1484 (1999)

5. Yang, ZW, Brunner, H: Quenching analysis for nonlinear Volterra integral equations. Dyn. Contin. Discrete Impuls. Syst., Ser. A Math. Anal. 27, 507-529 (2014)

6. Podlubny, I: Fractional Differential Equations. Academic Press, San Diego (1999)

7. Kirk, CM, Olmstead, WE: Thermal blow-up in a subdiffusive medium due to a nonlinear boundary flux. Fract. Calc. Appl. Anal. 17, 191-205 (2014)

8. Wyss, MM, Wyss, EW: Evolution, its fractional extension and generalization. Fract. Calc. Appl. Anal. 3, $273-284$ (2001)

9. Olmstead, WE, Roberts, CA: Thermal blow-up in a subdiffusive medium. SIAM J. Appl. Math. 69, $514-523$ (2008)

10. Yury, L: Maximum principle for the generalized time-diffusion equation. J. Math. Anal. Appl. 351, 218-223 (2009) 